International Journal of Pure and Applied Mathematics

Volume 85 No. $6 \quad 2013,983-992$

ISSN: 1311-8080 (printed version); ISSN: 1314-3395 (on-line version)

url: http://www.ijpam.eu

doi: http://dx.doi.org/10.12732/ijpam.v85i6.1

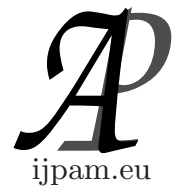

\title{
THE KRUSKAL PRINCIPLE
}

\author{
Yutaka Nishiyama \\ Department of Business Information \\ Faculty of Information Management \\ Osaka University of Economics
}

2, Osumi Higashiyodogawa Osaka, 533-8533, JAPAN

\begin{abstract}
Martin Kruskal invented an interesting card trick. After thoroughly shuffling and laying out a deck of cards, you use card values to determine how many cards to advance. Starting on the either the 1st or 2nd card in the series, you will end up at the same location with a very high probability. This can also be applied to Steve Humble'sgwalking game,h which I will describe as we use geometric distributions and Markov chains to calculate probabilities and further investigate this mystery.
\end{abstract}

AMS Subject Classification: 60J02, 97K02, 00A08

Key Words: Kruskal count, geometric distribution, Markov chain

\section{The Randomness Show}

A friend in the United Kingdom, Steve Humble, sent me a YouTube video of himself giving a presentation to some Irish mathematics teachers [1]. The video is called gDr. Maths Randomness Show, h and was staged in an Irish pub. Everyone looked like they were having a good time, but because of my limited English ability, it took a while before I figured out what they were talking about.

Received: December 10, 2012

(c) 2013 Academic Publications, Ltd. url: www.acadpubl.eu 
Here's what they did. First, Steve had a prepared deck of oversized playing cards, and passed them out to 8 members of the audience, so that each of them had 6 or 7 cards from the 52-card deck. They then laid out the cards in a random sequence on the floor, as shown in Figure 1. Since there are 52 cards, when you create 6 rows of 8 cards each you end up with 4 cards left over, so those become a shortened 7 th row.

Steve next had each of the 8 players stand on different cards in the 1 st row. Player 1 stood on the 9 of clubs (\$9), player 2 on the 6 of hearts (\$6), and so on, ending with player 8 on a 5 of diamonds $(\diamond 5)$.

Next, each player moved a number of cards equal to the face value of the card that they were standing on. Movement zigzagged back and forth, from left to right along the 1st row, right to left along the 2nd row, and so on. For example, player 1 was standing on $\mathbf{\beta} 9$, so that person started moving to the right. After advancing 7 cards to the right, the end of the row is reached; after descending to the next row, movement proceeds to the left, placing the player on the queen of diamonds $(\diamond \mathrm{Q})$. Face cards are taken to have a value of 1 , so when landing on one of them, you simply advance to the next card, in this case $\diamond 9$. Proceeding in this way, advancing as the cards instruct, results in a sequence like this:

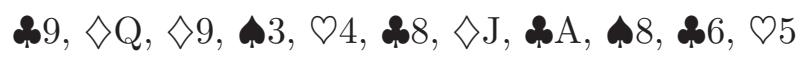

Here you to do this, you would end up at $\$ 5$, which instructs you to move 5 more cards, but because there's only 1 card remaining, you just stop there.

Steve explained these rules to the 8 players, and had each of them walk on the cards laid out on the floor (having player 8 start walking first and working backwards from there makes this easier to pull off). If 8 players standing on the 1st row of the cards shown in Figure 1 move according to these rules, here's how they would proceed:

\section{$* * *$}

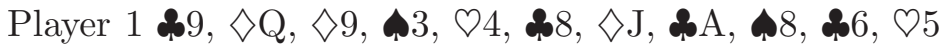

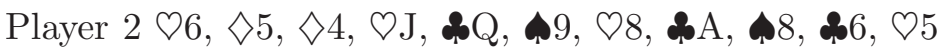

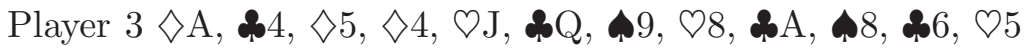

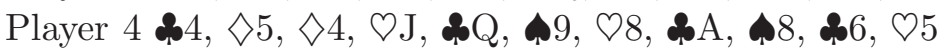

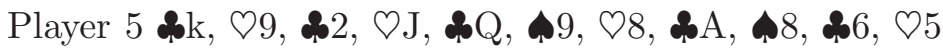

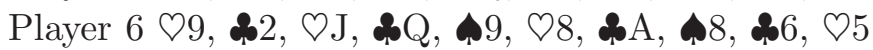

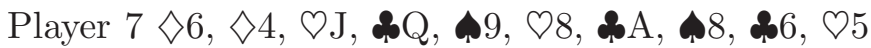

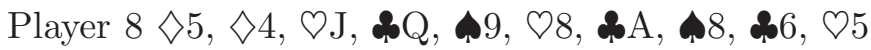




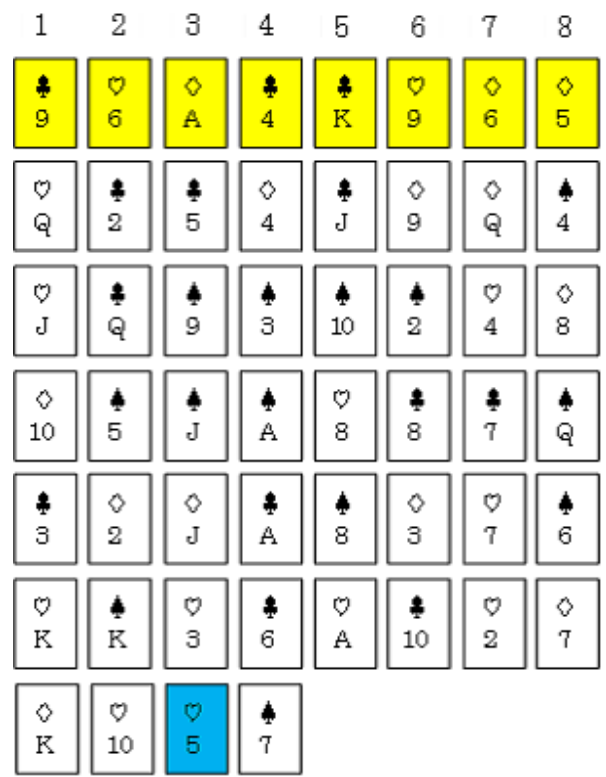

Figure 1: All 8 cards in the top row lead to the same destination.

Despite having started from different positions, as the players traverse the 52 cards each person ends up following a similar path, and in the end, each winds up on the $\mathrm{D} 5$. In the video Steve refers to this as eight strangers coming together and becoming new friends, a fun take on things.

\section{Our Paths Meet, $93 \%$ of the Time}

If you work out the math, it turns out that each player will arrive at the same card with a remarkably high probability of $93 \%$. We will now see how that happens, but before reading on I urge you to try this with your own deck of cards, to experience this odd phenomenon for yourself.

Figure 1 shows the case of 8 players, but to make the probability calculations a bit simpler, let's start with working out the odds for any given pair. We'll use the calculation method that Steve devised [2].

First, since the starting positions on cards 1-8 can be taken as an initial move of $1-8$, the average move was

$$
m=(1+2+3+\cdots+8) / 8=4.5 .
$$


The next move is according to the value shown on the card at the destination. That value will be 1 for an ace card, 2 for a 2 card, and so on through 10 , or 1 for a face card (jacks, queens, and kings). The average move is therefore

$$
m=(1+2+3+\cdots+10+1+1+1) / 13=4.46 .
$$

We let $p$ be the inverse of $m$, so we have $p=\frac{1}{m}$. This represents the probability of moving by the average move $m$, the significance of which we will discuss later. By using

$$
p=\frac{1}{4.46},
$$

let's calculate the probability of ending up at the same card. There are 52 cards in all, but subtracting from those a count equivalent to the average starting position (4.5), we consider there to be $52-4.5$ cards left. Proceeding from there, we will (on average) advance 4.46 cards with each move. This means that we can expect

$$
(52-4.5) / 4.46=10.65
$$

moves before ending. To make the calculations simpler, let's round that off to 10.

When 2 players make their initial selection of a starting point, the probability that they end up on the same card is $1 / 8$, so the probability that they start from different cards is $7 / 8$. With each move, the probability that both will end up on the same card is $p=\frac{1}{4.46}$, and the probability that they will not be on the same card is $1-p=1-\frac{1}{4.46}$. This means that the probability of remaining on a different card after both making an initial selection and moving 10 times is

$$
\left(\frac{7}{8}\right)\left(1-\frac{1}{4.46}\right)^{10} \approx 0.07
$$

The complementary event is therefore

$$
P=1-0.07=0.93
$$

In other words, while moving along the 52 cards the probability of both players landing on the same card at least once is 0.93 , or $93 \%$. I tested this theoretical probability using a computer-based simulation, and the results matched quite well.

Steve created Equation (1) as a rough estimation, but upon reflection there seems to be a slight problem with the left side. Since the initial selection of a 
value between 1 and 8 for the starting position represents an average $m=4.5$ advance, at this point $p$ is given by

$$
p=\frac{1}{m}=\frac{1}{4.5},
$$

so the left side should perhaps be

$$
\left(1-\frac{1}{4.5}\right)\left(1-\frac{1}{4.46}\right)^{10} \approx 0.06 .
$$

In any event, changing the equation does not result in a large difference in the results.

The calculations described above were for the case of 2 players ending up at the same location, but there were 8 players in the game shown in the video. Given that any pair of players will end up at the same location at least $93 \%$ of the time, we can predict that all 8 of them will end up on the same path with even higher probability.

\section{The Relation between Average $m$ and Probability $p$}

We can think of the relation between the average move number $m$ and the probability $p=1 / m$ as follows. If every card had a value of 1 , then the average move would be $m=1$ and the probability calculated from its inverse would obviously be $p=1 / m=1$.

$$
m=1, \quad p=\frac{1}{m}=1 .
$$

So let's consider the case where there are 7 values, $\{1,2,3,4,5,6,7\}$. These values are evenly distributed, so the average is

$$
m=(1+2+3+4+5+6+7) / 7=4,
$$

which lets us consider an average move as being an advance of 4 . In this case, the probability $p$ becomes

$$
p=\frac{1}{m}=\frac{1}{4} .
$$

If we take the probability of moving to location $\{i\}$ from among $\{1,2,3,4$, $5,6,7\}$ as $p_{i}$, then each probability will be equal.

$$
p_{1}=p_{2}=p_{3}=p_{4}=p_{5}=p_{6}=p_{7}=\frac{1}{7} \quad\left(\sum_{i=1}^{7} p_{i}=1\right) .
$$




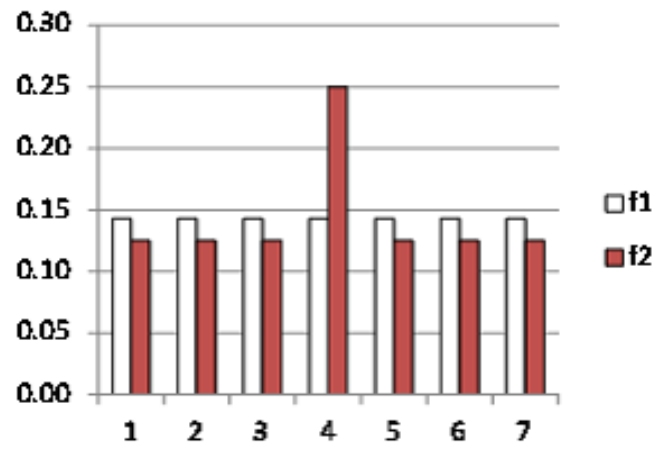

Figure 2: The normal probability distribution (f1) and the probability distribution using averages (f2)

The "f1" bars in Figure 2 show the probability distribution.

But how should we consider this probability when moving by average values? One good way is to think of four places that can be moved to, $\{4 \pm 0\}$, $\{4 \pm 1\},\{4 \pm 2\},\{4 \pm 3\}$, each with a probability $p=\frac{1}{4}$. If we take the probability of a move to $\{\mathrm{j}\}$ as $p\{j\}$, then we have

$$
p\{4 \pm 0\}=p\{4 \pm 1\}=p\{4 \pm 2\}=p\{4 \pm 3\}=\frac{1}{4}
$$

When we expand these to our 7 numbers, we get

$$
p_{1}=p_{2}=p_{3}=p_{5}=p_{6}=p_{7}=\frac{1}{8}, \quad p_{4}=\frac{1}{4} \quad\left(\sum_{i=1}^{7} p_{i}=1\right) .
$$

The "f2" bars in Figure 2 show this probability distribution.

The sum of the probabilities in $\mathrm{f} 1$ and $\mathrm{f} 2$ is 1 in both cases, despite the slightly different probability distributions of Equations $\left(^{*}\right)$ and $(* *)$. Below, we will use this gaveragesh approach to explain the probabilities.

If we use $\mu$ to represent the average of $\{1,2,3,4,5,6,7\}$ and $\sigma$ to represent the standard deviation, then we have

$$
\mu=4, \sigma=2 .
$$

We can use these values to find the normal distribution as follows (Figure 3):

$$
f(x)=\frac{1}{\sqrt{2 \pi} \sigma} e^{-\frac{1}{2 \sigma^{2}}(x-\mu)^{2}} \quad(-\infty<x<\infty) .
$$


When $x=4$, the probability becomes $f(4)=0.20$, which is close to the value of $p_{4}$ in Figure 2, but in a normal distribution the probability variable is defined as $-\infty<x<\infty$, resulting in quite a deviation from our probability distributions f1 and f2. We therefore will not use normal distributions.

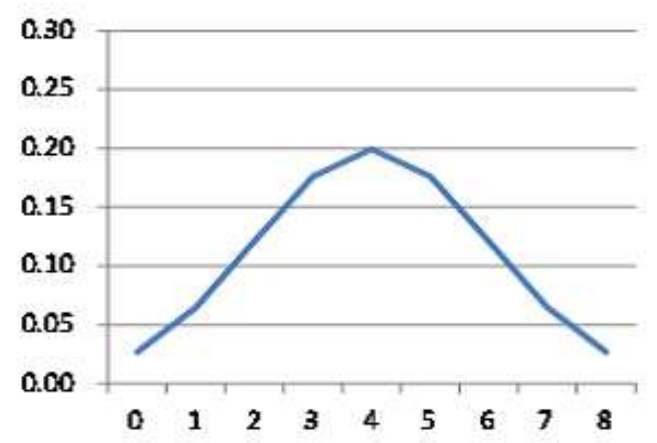

Figure 3: The normal distribution

\section{The Kruskal Principle}

Steve Humble's card trick, as it turns out, was an application of something called the Kruskal count, or sometimes the Kruskal principle. gKruskalh here refers to Martin Kruskal (1925 - 2006), who rediscovered this as a sideline to a body of other specialized research. It first appeared in an article "Sum Total" in the December 1957 issue of the magic periodical Ibidem by magician Alexander F. Kraus [3]. The first article that mentioned it was by Martin Gardner in Scientific American [4], but it has been discussed more recently by Julian Havil [5].

There's also a rigorous mathematical treatment in Jeffrey Lagarias' lengthy 22-page paper entitled gThe Kruskal Counth [6]. I would like to give an overview of the method for calculating probabilities presented there, as it differs slightly from Humble's method shown in Equation (1). We begin with the results.

As before, when we set the value of face cards as 1 , the average move becomes

$$
m=(1+2+3+\cdots+10+1+1+1) / 13=4.46,
$$


and the probability becomes

$$
p=\frac{1}{m}=\frac{1}{4.46}=0.22 .
$$

Then, according to Lagarias, the probability that the paths will meet somewhere within the $N(52)$ cards will be

$$
P=1-\left(1-p^{2}\right)^{N}=1-\left(1-\left(\frac{1}{4.46}\right)^{2}\right)^{52} \approx 0.931
$$

To help better understand Equation (3), I've prepared the two chains shown in Figure 4. These represent two decks of cards, and a progression among them according to independently read values. A stochastic process in which future behavior is determined by only a present value, with no influence from the past, is called a Markov chain. Looking at a current card value and advancing according to it is an excellent example. So chain 1 and chain 2 in the figure are two separate Markov chains.

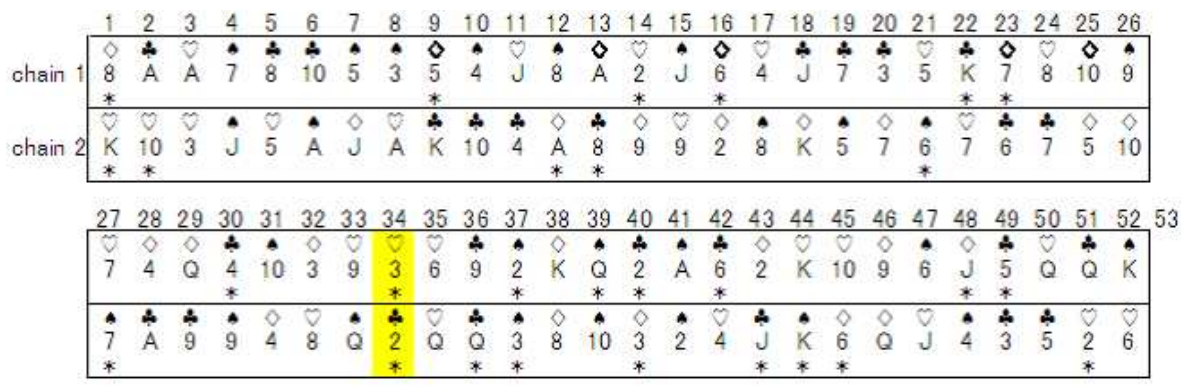

Figure 4: Correspondence between two chains

The 1 st card in chain 1 is $\diamond 8$, so you progress 8 cards to arrive at the 9 th card, $\diamond 5$. You then progress 5 cards to arrive at the 14 th, $\nabla 2$. Progressing along the sequence you get $\diamond 8, \diamond 5, \diamond 2, \diamond 6, \mathrm{c}$, and similarly progressing along chain 2 you get $\nabla \mathrm{K}, \nabla 10, \diamond \mathrm{A}$, $\mathbf{\$} 8$, c. The cards that you jump to after each move are shown with an asterisk. So, as the two chains progress through the 52 cards, what is the probability that they will, at some point, jump to cards that are in the same position within the chain? In the case of the chains in Figure 4, this happens at the 34th card.

Given the value of the 1st card, the probability of jumping to the 2nd card is $p$. The probability that both chain 1 and chain 2 will hit the 2 nd card, 
resulting in corresponding locations, is therefore

$$
p \times p=p^{2},
$$

and the probability that they will end up at different locations is the complementary value

$$
1-p^{2}
$$

It is known that the probability distribution of chain 1 and chain 2 not hitting corresponding locations in trials 1 through $i-1$ is a geometric distribution. In this problem, we thus want the sum of the probability of an initial correspondence on the 1st trial, plus the probability of an initial correspondence on the 2nd trial, and so on through the probability of an initial correspondence on the $N$ th trial (the integral of a geometric progression), but the calculation is quite simple if we consider complementary events.

We do so by finding the probability of chain 1 and chain 2 not corresponding after the 1st trial, and not corresponding after the 2nd trial, and so on through not corresponding on the $N$ th (52nd) trial. That is simply

$$
\left(1-p^{2}\right)^{N}
$$

The probability that an initial correspondence occurs at least once is the complementary event of the above, which is calculated as

$$
P=1-\left(1-p^{2}\right)^{N}
$$

This is where Lagarias'Equation (3) comes from.

Applying this to Humble's method of Equation (1), we get

$$
P=1-(1-p)^{\frac{N}{m}}=1-\left(1-\frac{1}{4.46}\right)^{\frac{52}{4.46}} \approx 0.948 .
$$

Here, $m$ is the average move, and $p=1 / m$. Taking the total number of cards as $N$ and dividing by $m$, we get the number of card reads and jumps. This is represented in Figure 4 by the number of asterisks, which is approximately 12. The probability that the two chains will hit corresponding locations while advancing on average $m$ cards is $p$. The probability that no correspondence will occur is the complement of that, $(1-p)$, and Equation (4) shows the complementary event of having no correspondence between jumps among all the jumps performed. 
At first glance, Equations (3) and (4) appear to be different, but they are in fact the same probability as found through different approaches, and inserting the same value into each will give the same result.

The Kruskal principle also has connections with ergodic theory, which says that the space average in a stochastic process is equivalent to the time average. Ergodic theory came about through attempts to probabilistically understand the movement of molecules in a gas, so it's interesting to see such deep theoretical underpinnings to what on the surface appears to be a simple magic trick. I hope you will pick up a book on the subject if you are interested in learning more.

\section{References}

[1] S. Humble, Dr. Maths Randomness Show, Alchemist Cafe, Dublin (2010), http://www.youtube.com/watch?v=goIOWcnagP0

[2] S. Humble, Magic Card Maths, The Montana Mathematics Enthusiast, 5, No-s: 2, 3, 327-336 (2008).

[3] A.F. Kraus, Sum Total, Ibidem, 232, No. 12 (1957); No. 13 (1958), 255259.

[4] M. Gardner, Mathematical Games, Scientific American, 238, No. 2 (1978).

[5] J. Havil, Impossible?: Surprising Solutions to Counterintuitive Conundrums, Princeton University Press (2008).

[6] J.C. Lagarias, E. Rains, R.J. Vanderbei, The Kruskal Count, Cornell University Library (2001). 